\title{
Cisão de Cadeia na Degradação Termo-Mecânica do Poliestireno sob Múltiplas Extrusões
}

\author{
Carlos A. Cáceres \\ Programa de Pós-Graduação em Ciência e Engenharia de Materiais, UFSCar \\ Sebastião V. Canevarolo \\ Departamento de Engenharia de Materiais, UFSCar
}

\begin{abstract}
Resumo: Determinou-se o número de cisões de cadeia gerado pela degradação termo-mecânica do poliestireno quando submetido a múltiplas extrusões. A degradação foi acompanhada pelas mudanças nas curvas de distribuição de massa molar. Seguindo-se o significado físico das massas molares médias de uma curva de MWD mostrou-se que a massa molar numérica media $\overline{\mathrm{M}}_{n}$ é a única que pode ser relacionada diretamente com o número de moléculas do sistema. A partir desta calculou-se o número de cadeias clivadas (ns) como uma relação entre a $\overline{\mathrm{M}}_{\mathrm{n}}$ da amostra degradada e a virgem. A função de distribuição de cisão de cadeia (CSDF) mostra que o processo de degradação termo-mecânica do poliestireno submetido a múltiplas extrusões à $240^{\circ} \mathrm{C}$ é do tipo aleatório, independente da massa molar inicial.
\end{abstract}

Palavras-chave: Degradação termo-mecânica, extrusão, poliestireno, cisão de cadeia.

\section{Chain Scission in the Thermo Mechanical Degradation of Polystyrene under Multiple Extrusions}

Abstract: The number of chain scissions during thermo-degradation of polystyrene under multiple extrusions was calculated. The degradation produces changes in the molecular weight distribution MWD curves. Following the physical meaning of the average molecular weights of a MWD curve it was shown that the number average molecular weight $\bar{M}_{n}$ is the only average that can be directly related to the number of molecules in the system. From that it was calculated the number of sectioned chains $(n s)$ as a ratio between the number of chains in the degraded and original polymers. The chain scission distribution function (CSDF) shows that the thermo-mechanical degradation process of polystyrene under multiple extrusions at $240{ }^{\circ} \mathrm{C}$ is of a random type, independent of the initial molecular weight.

Keywords: Thermo-mechanical degradation, extrusion, polystyrene, chain scission.

\section{Introdução}

Durante o processo de propagação da cadeia radical na polimerização em cadeia os centro ativos da frente em crescimento se instabilizam e morrem aleatoriamente, o qual gera cadeias poliméricas com diferentes tamanhos, formando uma distribuição de tamanhos em torno de um valor médio, o que exige uma curva de distribuição de massa molar (MWD) para representá-lo. A partir de $1964^{[1]}$ a cromatografia de exclusão por tamanho (SEC) tem sido extensivamente utilizada na caracterização de polímeros, através da obtenção das curvas de distribuição de massa molar (MWD). A análise das curvas de MWD pode ajudar no entendimento das propriedades físico-químicas dos polímeros incluindose a elucidação dos processos de degradação termo-mecânica. Infelizmente a análise destas curvas é ainda difícil e não está completamente equacionada. Assim a maioria das publicações se restringe a analise das mudanças nas massas molares médias, não explorando apropriadamente todas as informações contidas em uma curva de MWD.

Para melhor entender os processos de degradação que ocorrem durante o processamento termo-mecânico de um polímero foi proposta uma metodologia de cálculo ${ }^{[2,3]}$ que permite quantificar o deslocamento total da curva de MWD de uma amostra degradada com relação à amostra virgem, não se limitando a calcular apenas a alteração de seus valores médios. Essa metodologia é chamada de função de distribuição de cisão de cadeia (CSDF) e foi utilizada para acompanhar a degradação termo-mecânica do polipropileno ${ }^{[2-5]} \mathrm{e}$ polietileno de alta densidade ${ }^{[6,7]}$ durante a extrusão.

$\mathrm{Na}$ literatura dos anos $70^{[8,9]}$ pode-se encontrar uma equação que utiliza a massa molar numérica media $\left(\overline{\mathrm{M}}_{\mathrm{n}}\right)$ da amostra antes e após ter sofrido degradação, que segundo os autores, permite calcular o número de cisões de cadeia, e que vem sendo utilizada freqüentemente na literatura ${ }^{[10-15]}$. Pode-se também encontrar outras equações que utilizam relações entre

Autor para correspondência: Sebastião V. Canevarolo, Departamento de Engenharia de Materiais, UFSCar, Rod. Washington Luís, Km 235, CEP: 13560-905, São Carlos, SP, Brasil. E-mail: caneva@ufscar.br 
outras massas molares medias $\left(\overline{\mathrm{M}}_{\mathrm{n}}, \overline{\mathrm{M}}_{\mathrm{w}}\right.$ e $\left.\overline{\mathrm{M}}_{\mathrm{z}}\right)$ para quantificar a intensidade da degradação. Inclusive é sugerido ser possível calcular o número de cisões de cadeia e/ou entrecruzamento a partir da relação entre diferentes massas molares médias ${ }^{[16]}$. Ao se fazer tais generalizações não se está sendo suficientemente cuidadoso, já que devemos levar em consideração o significado físico e a derivação matemática das diferentes formas de se calcular cada uma das médias sugeridas, e que portanto elas não devem ser vistas simplesmente como valores médios.

No caso específico do poliestireno a cisão de cadeia durante a oxidação térmica pode ser atribuída à decomposição de radicais alcoxi terciários, onde as reações podem ocorrer de forma similar à degradação do PP, ou por cisão homolítica de grupos hidroperóxidos. Durante o processamento do PS a massa molar diminui mesmo se a quantidade de oxigênio é limitada. A degradação final é resultado da degradação termomecânica causada pelo cisalhamento e a degradação oxidativa, limitada à quantidade de oxigênio presente. Para melhor entender a degradação térmica do PS considera-se a presença de ligações lábeis. A degradação térmica depende fortemente da natureza dos grupos terminais das cadeias e estes podem variar. Grupos terminais de cadeia benzênica geram cisão de ligações C-C que por desproporção dos radicais formados produzem tolueno e terminais de cadeia insaturadas. Neste caso considera-se que os radicais iniciam os processos de volatilização ${ }^{[17]}$. A degradação térmica do poliestireno tem um comportamento intermediário entre a despolimerização e a cisão de cadeia aleatória. Isto se deve ao fato que a reação de despolimerização cessa antes de desfazer completamente a cadeia polimérica. Degradação térmica do PS a altas temperaturas, entre 360 e $420{ }^{\circ} \mathrm{C}$, produz uma forte despolimerização com produção de $40 \%$ de estireno, $2,4 \%$ de tolueno e outros produtos com massa molar média de $264^{[18]}$.

Por outro lado Gupta et al. ${ }^{[19]}$ notaram que a degradação térmica do PS quando realizada a baixas temperaturas, entre 205 e $255^{\circ} \mathrm{C}$, pode ocorrer por cisão aleatória de cadeia e despolimerização na presença do oxigênio do ar. Os autores indicaram que existe um tempo inicial para o começo da degradação seguido de uma rápida queda da massa molar, atribuída à presença de ligações lábeis, sendo que só após algum tempo inicia-se a despolimerização. A redução da taxa de cisão de cadeia com o tempo pode ser interpretada devido à afinidade do oxigênio pelos radicais, desta forma diminuindo a freqüência de transferência das cadeias terminais termolábeis. O oxigênio tem um efeito duplo tanto como iniciador da cisão das cadeias terminais como também pela afinidade com radicais, fazendo diminuir a freqüência da reação de transferência. Este estudo também mostra, através da análise termogravimétrica, que a decomposição térmica do PS apresenta uma perda em massa de $\cong 10 \%$ após 30 minutos à temperatura de $240{ }^{\circ} \mathrm{C}$. Este tempo é muito maior que os tempos de residência normalmente observados em uma extrusora. Neste trabalho o tempo médio de residência na extrusora W\&P ZSK 30 é de aproximadamente 1,5 minuto e o tempo máximo não ultrapassa 4 minutos.
Peterson et al. ${ }^{[20]}$ estudaram a degradação térmica do poliestireno através da termogravimetria e calorimetria diferencial de varredura (DSC) sob atmosferas de nitrogênio e ar. Os autores observaram que a energia de ativação do PS sob atmos-

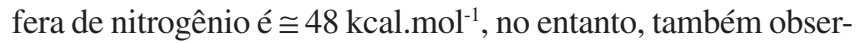
varam valores da energia de $\cong 36 \mathrm{kcal} . \mathrm{mol}^{-1}$ no estágio inicial da degradação e sugeriram que a degradação é iniciada por ligações lábeis. Em atmosfera de ar a degradação ocorre via reações que envolvem decomposição de peróxidos e exibem uma energia de ativação de $\cong 30 \mathrm{kcal}^{\mathrm{mol}} \mathrm{l}^{-1}$. Curvas termogravimétricas indicam que a degradação do PS ocorre em uma só via de $250-400{ }^{\circ} \mathrm{C}$ e observando essa curva de TG percebe-se que a perda de massa abaixo de $250{ }^{\circ} \mathrm{C}$ é menor que $1 \%$.

$\mathrm{Na}$ parte inicial deste trabalho apresentamos um desenvolvimento matemático para calcular o número de cadeias clivadas, que em conjunto com a aplicação da CSDF, será usado na parte final para quantificar a degradação termo-mecânica do poliestireno sob múltiplas extrusões a $240^{\circ} \mathrm{C}$.

\section{Teoria}

O número de cadeias clivadas (ns), que ocorre em um processo de degradação, pode ser calculado a partir da diferença entre o número de cadeias (ou moléculas) antes e após a degradação, representada pela Equação 1.

$$
n_{S}=N_{T(D)}-N_{T(O)}
$$

Conseqüentemente, pode-se estimar a fração de cadeias clivadas $(f s)$ como mostrado pela Equação 2.

$$
f_{S}=\frac{N_{T(D)}-N_{T(O)}}{N_{T(O)}}
$$

sendo $\mathrm{N}_{\mathrm{T}}$ o número de cadeias ou moléculas e os subíndices (D) e $(\mathrm{O})$ indicadores da amostra ser degradada ou original (ou referência), respectivamente.

As massas molares médias são calculadas a partir de relações entre os momentos de uma curva de distribuição de massa molar (MWD). A Equação 3 expressa matematicamente como calcular qualquer massa molar média, onde $\alpha$ é o enésimo momento de uma curva de distribuição de massa molar, $\mathrm{N}$ é o número de moléculas e $\mathrm{M}$ é a massa molar. A média é chamada de Massa Molar Numérica Média $\left(\bar{M}_{\mathrm{n}}\right)$ quando $\mathrm{k}=\mathrm{n}$ e $\alpha=1$, Massa Molar Ponderal Média $\left(\overline{\mathrm{M}}_{\mathrm{w}}\right)$ quando $\mathrm{k}=\mathrm{w}$ e $\alpha=2$ e Massa Molar Z-média $\left(\overline{\mathrm{M}}_{\mathrm{z}}\right)$ quando $\mathrm{k}=\mathrm{z}$ e $\alpha=3$.

$$
\bar{M}_{k}=\frac{\sum N_{i} M_{i}^{\alpha}}{\sum N_{i} M_{i}^{\alpha-1}}
$$

Se o peso da amostra é normalizado $\left(\Sigma \mathrm{N}_{\mathrm{i}} \mathrm{M}_{\mathrm{i}}=1\right)$ a uma constante, podemos relacionar diretamente a massa molar numérica média com o número de moléculas, ou seja $\Sigma \mathrm{N}_{\mathrm{i}}=\mathrm{N}_{\mathrm{t}}$. Portanto essa relação é exclusiva da $\overline{\mathrm{M}}_{\mathrm{n}}$ e não de nenhuma outra massa molar média de ordem superior. 


\section{Número $\left(n_{s}\right)$ e fração $\left(f_{s}\right)$ de cadeias clivadas}

A massa molar numérica média $\overline{\mathrm{M}}_{\mathrm{n}}$, obtida fazendo $\alpha=1$ na Equação 3, nada mais é que a normalização da massa da amostra pelo número total de moléculas contida nesta amostra, segundo a Equação 4:

$$
\begin{array}{r}
\bar{M}_{n}=\frac{\sum N_{i} M_{i}}{\sum N_{i}}=\frac{\sum W_{i}}{\sum N_{i}}=\frac{W_{T}}{N_{T}}= \\
\text { Massa total da amostra }
\end{array}
$$

\section{Número total de moléculas na amostra}

Assim em um sistema polidisperso é possível, partindo-se da Equação 4, calcular o número de moléculas presente $\mathrm{N}_{\mathrm{T}}$ conhecendo-se sua massa molar numérica média segundo a Equação 5:

$$
N_{T}=\frac{W_{T}}{\bar{M}_{n}}
$$

Se assumirmos que durante a degradação termo-mecânica a perda de massa para a formação de voláteis é desprezível (coletou-se $10 \mathrm{mg}$ na degasagem para $1 \mathrm{~kg}$ de PS extrudado equivalendo a $0,001 \% \mathrm{w}$ ) e sabendo-se que durante a obtenção das curvas de SEC o valor da massa de amostra analisado é normalizado e constante, podemos assumir como válida a conservação de massa antes a depois da degradação. Isto é descrito na Equação 6:

$$
\sum W_{i}=W_{T(O)}=W_{T(D)}
$$

Tendo em mente a Equação 6 e partindo-se da Equação 5 e substituindo-a na Equação 1 obtem-se o número de cisões de cadeia em um mol de moléculas, que multiplicado pelo número de Avogadro fornece o número de cadeias clivadas $\left(\mathrm{n}_{\mathrm{s}}\right)$ por grama de polímero, como mostra a Equação 7.

$$
n_{s}=W_{T} N_{A}\left(\frac{1}{\bar{M}_{n(D)}}-\frac{1}{\bar{M}_{n(O)}}\right)
$$

sendo $\mathrm{W}_{\mathrm{T}}$ a massa total, $\mathrm{N}_{\mathrm{A}}$ o número de Avogadro e $\overline{\mathrm{M}}_{\mathrm{n}}$ a massa molar numérica média com os subíndices (D) e (O) indicando ser a amostra degradada e original (ou referência), respectivamente.

Por outro lado se a Equação 5 for substituída na Equação 2 obtem-se a fração de cadeias clivadas (f), expressa pela Equação 8.

$$
f_{s}=\frac{\bar{M}_{n(O)}}{\bar{M}_{n(D)}}-1
$$

\section{Experimental}

\section{Material}

O polímero utilizado foi um poliestireno cristal comercial (Polystyrol 168M) fornecido pela BASF S.A. O material foi utilizado como recebido, não sendo adicionado nenhum ou- tro tipo de estabilizante. Este tem densidade de $1,04 \mathrm{~g} . \mathrm{cm}^{-3} \mathrm{e}$ MFI de 1,5 g/10 min, medido a $200{ }^{\circ} \mathrm{C}$ e $5,0 \mathrm{~kg}$, seguindo a norma ASTM-D1238.

\section{Extrusão}

O poliestireno foi processado em uma extrusora dupla rosca modular, do tipo interpenetrante, co-rotativa Werner \& Pfleiderer ZSK-30, equipada com um alimentador gravimétrico da K-Tron. Os parâmetros operacionais foram mantidos constantes com uma taxa de alimentação de $5 \mathrm{~kg} / \mathrm{h}$, velocidade de rotação da rosca de 100 minutos, perfil de rosca do tipo $2 \mathrm{~KB} 45^{[6]}$ e um perfil constante de temperatura de $240{ }^{\circ} \mathrm{C}$ ao longo do barril da extrusora. O polímero foi reprocessado por até cinco vezes, coletando-se quantidade suficiente de amostra a cada extrusão para posterior análise.

\section{Reometria}

As curvas de viscosidade em função da taxa de cisalhamento (50 a $5000 \mathrm{~s}^{-1}$ ) foram obtidas em um reometro capilar Instron 4467, a $240{ }^{\circ} \mathrm{C}$ a mesma utilizada durante o processo de extrusão. A conversão de carga e velocidade do pistão para tensão e taxa de cisalhamento foi realizada pelo programa Rheo Soft.

\section{Cromatografia de exclusão por tamanho}

A análise de cromatografia de exclusão por tamanho foi realizada em um cromatógrafo Waters modelo $150 \mathrm{CV}$ com detector por índice de refração. Utilizou-se quatro colunas HT (Styragel) calibradas com 16 padrões de poliestireno da Shodex Standard na faixa de $5 \times 10^{2}$ a $1 \times 10^{6}$ Dalton. As amostras foram solubilizadas por uma hora à temperatura de $140{ }^{\circ} \mathrm{C}$ em 1, 2, 4 Triclorobenzeno (grau HPLC), com antioxidante Irganox 1010, filtradas e $200 \mu \mathrm{L}$ foi injetado no cromatógrafo operando a temperatura de $135^{\circ} \mathrm{C}$ e vazão de $1 \mathrm{~mL} / \mathrm{min}$. As curva de distribuição de massa molar MWD foram calculadas utilizando-se o programa Millennium e exportadas como arquivos ASCII.

\section{Função de distribuição de cisão de cadeia (CSDF)}

Os arquivos ASCII foram re-apresentados no software Microsoft Excel ${ }^{\circledR}$ e as curvas da Função de Distribuição de Cisão de Cadeia (CSDF) foram calculadas utilizando-se o programa CSDF 4.1 ${ }^{[3]}$, disponível a quem se interessar.

\section{Resultados e Discussão}

\section{Número de cadeias clivadas}

Na Tabela 1 são mostradas as massa molares médias $\left(\overline{\mathrm{M}}_{\mathrm{n}}, \overline{\mathrm{M}}_{\mathrm{w}}, \overline{\mathrm{M}}_{\mathrm{z}}\right)$, polidispersão de primeira e segunda ordem $\left(\overline{\mathrm{M}}_{\mathrm{w}} / \overline{\mathrm{M}}_{\mathrm{n}}, \overline{\mathrm{M}}_{\mathrm{z}} / \overline{\mathrm{M}}_{\mathrm{w}}\right)$, número $\left(\mathrm{n}_{\mathrm{s}}\right)$ e fração (f $\left.\mathrm{f}_{\mathrm{s}}\right)$ de cadeias clivadas. Os resultados mostram que todas as massas molares apresentam uma redução similar e conseqüentemente os índices de polidispersão permanecem praticamente constantes $(\mathrm{IP} \cong 2)$. A partir da primeira até a terceira extrusão o número 
Tabela 1. Massas molares médias $\left(\overline{\mathrm{M}}_{\mathrm{n}}, \overline{\mathrm{M}}_{\mathrm{w}}, \overline{\mathrm{M}}_{\mathrm{z}}\right)$, índices de polidispersão $\left(\overline{\mathrm{M}}_{\mathrm{w}} / \overline{\mathrm{M}}_{\mathrm{n}}, \overline{\mathrm{M}}_{\mathrm{z}} / \overline{\mathrm{M}}_{\mathrm{w}}\right)$, número (n $)$ e fração (f $)$ de cadeias clivadas.

\begin{tabular}{cccccccc}
\hline $\begin{array}{c}\mathbf{N}^{\mathbf{0}} \\
\text { Extrusões }\end{array}$ & $\overline{\mathbf{M}}_{\mathrm{n}}$ & $\overline{\mathbf{M}}_{\mathrm{w}}$ & $\overline{\mathbf{M}}_{\mathrm{z}}$ & $\overline{\mathbf{M}}_{\mathrm{w}} / \overline{\mathbf{M}}_{\mathrm{n}}$ & $\overline{\mathbf{M}}_{\mathrm{z}} / \overline{\mathbf{M}}_{\mathrm{w}}$ & $\mathbf{n}_{\mathrm{s}}$ & $\mathbf{f}_{\mathrm{s}}$ \\
\hline PS* & 212.000 & 379.000 & 630.000 & 1,79 & 1,66 & - & - \\
PS x 1 & 196.000 & 363.000 & 610.000 & 1,85 & 1,68 & $2,32 \mathrm{E}+17$ & 0,08 \\
PS x 2 & 202.000 & 358.000 & 580.000 & 1,77 & 1,62 & $1,41 \mathrm{E}+17$ & 0,05 \\
PS x 3 & 182.000 & 322.000 & 527.000 & 1,77 & 1,64 & $4,68 \mathrm{E}+17$ & 0,16 \\
PS x 4 & 144.000 & 254.000 & 406.000 & 1,76 & 1,60 & $1,34 \mathrm{E}+18$ & 0,47 \\
PS x 5 & 120.000 & 210.000 & 330.000 & 1,75 & 1,57 & $2,18 \mathrm{E}+18$ & 0,77 \\
\hline
\end{tabular}

*Amostra de PS como recebido.

de cadeias clivadas por grama de amostra $\left(\mathrm{n}_{\mathrm{s}}\right)$ esta na ordem de $10^{17}$ e nas extrusões seguintes (x 4 e x 5) este valor aumenta para $10^{18}$ cadeias clivadas por grama de PS. A fração de cadeias clivadas ( $\left.f_{s}\right)$ indica a tendência de aumentar o número de cisões de cadeia com o número de extrusões, atingindo na quarta extrusão uma cadeia clivada para cada duas cadeias originais $\left(\mathrm{f}_{\mathrm{s}}=0,47\right)$. Já na quinta extrusão um total de quase $80 \%\left(f_{s}=0,77\right)$ das cadeias originais sofreram cisão.

\section{Distribuição de massa molar e reologia}

A Figura 1 mostra as curvas de distribuição de massas molares MWD do poliestireno como recebido e após os vários reprocessamentos a $240{ }^{\circ} \mathrm{C}$. Pode-se notar que estas curvas se deslocam no sentido da redução da massa molar, sem alterar a sua forma, ou seja, tem-se redução nas massas molares médias mas a polidispersão se mantem constante, como já observado pelos dados da Tabela 1 .

A Figura 2 mostra as curvas da viscosidade em função da taxa de cisalhamento ( $\left.\cong 50 \mathrm{a} 7000 \mathrm{~s}^{-1}\right)$ do poliestireno virgem e após múltiplas extrusões. Nas menores taxas de cisalhamento a viscosidade é dependente do nível de degradação termomecânica imposto, sendo menor quanto maior o número de extrusões e esta diferença diminui com o aumento da taxa de cisalhamento. Este comportamento é reflexo das mudanças que ocorreram nas curvas de MWD, ou seja, redução da massa molar ponderal com o número de extrusões e manutenção

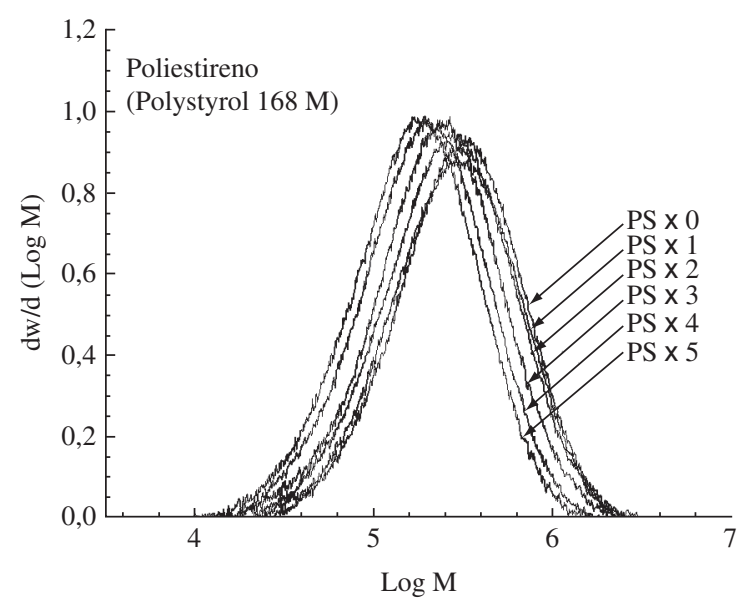

Figura 1. Curvas de distribuição de massa molar do poliestireno como recebido (PS x 0) e após múltiplas extrusões (PS x 1 - PS x 5) a $240{ }^{\circ} \mathrm{C}$.

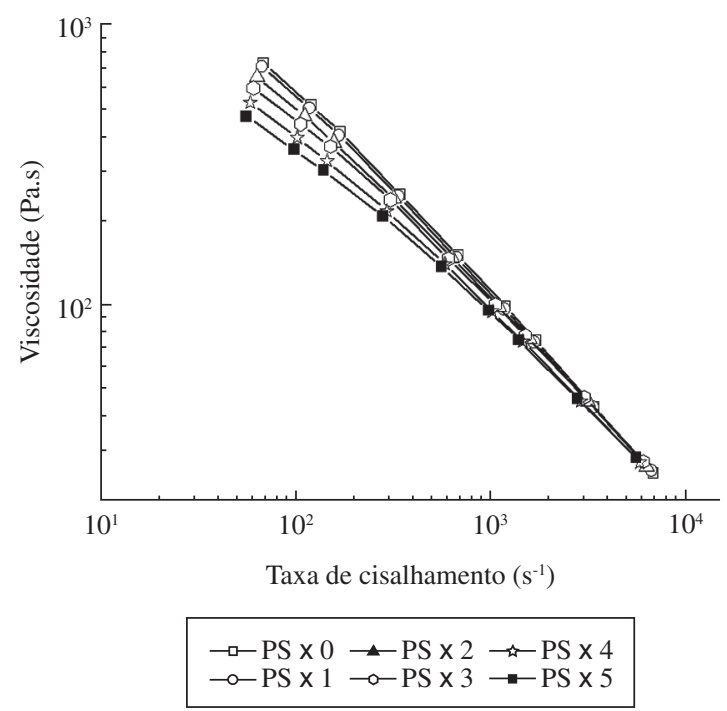

Figura 2. Curvas da viscosidade a $240{ }^{\circ} \mathrm{C}$ em função da taxa de cisalhamento para o poliestireno como recebido (PS x 0) e após múltiplas extrusões (PS $\times 1$ - PS x 5).

da polidispersão $\left(\bar{M}_{w} / \bar{M}_{n} \cong 2\right)$ pois a viscosidade nas baixas taxas de cisalhamento é afetada pela massa molar ponderal média, e nas altas taxas de cisalhamento é dependente da largura da MWD, expressa através da polidispersão $\mathrm{o}^{[21]}$.

\section{Distribuição de cisão de cadeia (CSDF)}

A Figura 3 mostra as curvas de CSDF, calculadas e apresentadas em função da massa molar inicial, para o poliestireno sujeito a múltiplas extrusões a $240{ }^{\circ} \mathrm{C}$. As curvas de CDSF são principalmente linhas retas sem inclinação que se deslocam para cima com o aumento do número de extrusões. Assim os maiores valores são característicos das amostras da quinta extrusão. Estes resultados indicam que o processo de degradação termo-mecânica do poliestireno sob múltiplas extrusões reduz gradativamente a massa molar das cadeias, independentemente de seu comprimento (ou massa molar). Já é conhecido ${ }^{[17-20]}$ que a degradação térmica do poliestireno a baixas temperaturas gera um mecanismo de cisão de cadeia aleatória, independente da massa molar da cadeia que está sendo degradada. Os dados deste trabalho mostram que este mecanismo também acontece na presença de cisalhamento, ou seja a degradação termo-mecânico também ocasiona a cisão das cadeias mas ela é indepen- 


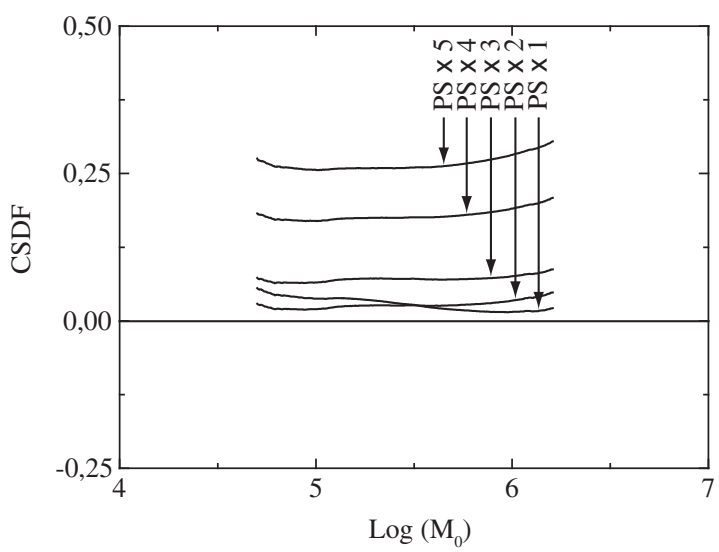

Figura 3. Curvas da função de distribuição de cisão de cadeia (CSDF) do poliestireno degradado sob múltiplas extrusões a $240{ }^{\circ} \mathrm{C}$, em função da massa molar inicial (PS x 0).

dente de seu comprimento inicial, indicando que o processo de degradação termo-mecânico do PS é do tipo aleatório.

\section{Conclusões}

Neste trabalho apresenta-se uma metodologia para calcular o número de cadeias clivadas. Aplicando-se este conceito ao poliestireno submetido à degradação termo-mecânica na forma de um processamento sob múltiplas extrusões a $240{ }^{\circ} \mathrm{C}$ mostrou-se que o número de cadeias clivadas por grama de PS $n_{\mathrm{s}}$ está na faixa de $10^{17}$ a $10^{18}$ cadeias para cada extrusão. A fração de cadeias clivadas aumenta com o número de extrusões, tendo a relação de uma cadeia clivada para cada duas iniciais $\left(f_{s} \cong 1 / 2\right)$ na quarta extrusão e quase quatro cadeias de cinco originais $\left(f_{s}=0,77\right)$ são clivadas na quinta extrusão. As curvas de CSDF mostram-se como linhas retas sem inclinação, aumentando de valor com o número de extrusões e independentes da massa molar inicial. Estes resultados indicam que o processo de degradação termo-mecânico do poliestireno sob múltiplas extrusões é um processo do tipo aleatório. O número de cisões de cadeia é um dado importante na simulação de processos de degradação, portanto acredita-se que a forma de calcular proposta neste trabalho possa ser de grande valia para subsidiar estas simulações.

\section{Agradecimentos}

Os autores agradecem ao $\mathrm{CNPq} / \mathrm{FINEP}$ pelo apoio financeiro em vários projetos. C.A.C.C. agradece à CAPES/CNPq pela bolsa de estudos.

\section{Referências Bibliográficas}

1. Moore, J. C. - J. Polym. Sci. Pt. A, 2, p.835, (1964).

2. Canevarolo, S. V. - Polym. Degrad. Stab., 70, (1), p.71, (2000).
3. Cáceres, C. A. \& Canevarolo, S. V. - Polym. Degrad. Stab., 86, (3), p.437, (2004).

4. Cáceres, C. A. \& Canevarolo, S. V. - Polimeros: Cienc Tecnol, 16, (4), p.294, (2006).

5. Machado, A.; Maia, J.; Canevarolo, S. V. \& Covas, J. Apply. Polym. Sci., 91, (4), p.2711, (2004).

6. Pinheiro, L. A.; Chinelatto, M. A. \& Canevarolo, S. V. Polym. Degrad. Stab., 86, (3), p.445, (2004).

7. Pinheiro, L. A.; Chinelatto, M. A. \& Canevarolo, S. V. Polym. Degrad. Stab., 91, (10), p.2324, (2006).

8. Reich, L. \& Stivala, S. S. - "Elements of polymer degradation", McGraw-Hill, New York (1971).

9. Tobolsky, A. V.; Kotliar, A. M. \& Lee, T. C. P. - "Thermal Stability of polymer", in: Random scission process, cap. 4, Conley, R. T. (ed.), Marcel Dekker Inc., New York (1970).

10. Chan, J. H. \& Balke, S. T. - Polym. Degrad. Stab., 57, (2), p.113, (1997).

11. El'Darov, E. G.; Mamedov, F. V.; Gol'dberg, V. M. \& Zaikov, G. E. - Polym. Degrad. Stab., 51, (3), p.271, (1996).

12. Dong, D.; Tasaka, S. \& Inagaki, N. - Polym. Degrad. Stab., 72, (2), p.345, (2001).

13. David, C.; Trojan, M.; Daro, A. \& Demarteau, W. Polym. Degrad. Stab., 37, (2), p.233, (1992).

14. Chiantore, O.; Tripodi, S.; Sarmoria, C. \& Vallés, E. Polym., 42, (9), p.3981, (2001).

15. Bottino, F. A.; Cinquegrani, A. R.; Di Pascuale, G.; Leonardi, L.; Orestano, A. \& Pollicino, A. - Polym. Test., 23, (7), p.779, (2004).

16. Shyichuk, A. V. \& Lutsjak, V. S. - Eur. Polym. J., 31, (7), p.631, (1995).

17. Gugumus, F. - "Oxidation Inhibition in Organic Materials", in: Stabilization of Plastics Against Thermal Oxidation, cap. 4, Pospíšil, J. \& Klemchuk, P. P. (ed.), CRC Press, Boca Raton (1990).

18. Billmeyer, F. W. - "Textbook of Polymer Science”, John Wiley, New York (1975).

19. Gupta, M. C. \& Nath, J. D. - J. Appl. Polym. Sci., 25, p.1017, (1980).

20. Peterson, J. D.; Vyazovkin, S. \& Wight, C. A. - Macromol. Chem. Phys., 202, p.775, (2001).

21. Han, C. D. - "Rheology in Polymer Processing", Academic Press, New York (1976).

Enviado: $27 / 12 / 07$

Reenviado: $11 / 06 / 08$

Aceito: $23 / 06 / 08$ 